\title{
Simultaneous Segmentation and Registration for Medical Image
}

\author{
Chen Xiaohua ${ }^{1}$, Michael Brady ${ }^{1}$, and Daniel Rueckert ${ }^{2}$ \\ 1 Robotics Research Group, University of Oxford, Oxford, UK \\ $\{$ xchen, jmb\}@robots.ox.ac.uk \\ 2 Department of Computing, Imperial College London, London, UK
}

\begin{abstract}
Although segmentation and registration are usually considered separately in medical image analysis, they can obviously benefit a great deal from each other. In this paper, we propose a novel scheme of simultaneously solving for segmentation and registration. This is achieved by a maximum a posteriori (MAP) model. The key idea is to introduce an additional hidden Markov random vector field into the model. Both rigid and non-rigid registration have been incorporated. We have used a B-spline based free-form deformation for non-rigid registration case. The method has been applied to the segmentation and registration of brain MR images.
\end{abstract}

\section{Introduction}

The large number of segmentation and registration methods found in the literature shows that these are two of the most studied topics in medical image analysis. However, most existing work considers them separately, even though they are closely related: the solution of one can greatly assist in the computation of the other. Using segmentation results can reduce the influence of noise on the original images and lead to improved registration. In the case that one image scan presents rather subtle information about the subject to be segmented, significant improvement may be obtained by combining information from images of the same subject acquired at different times or under different conditions. In order to use this information, images need to be perfectly aligned.

Until recently, little research has been done to simultaneously estimate the segmentation and registration problems in a single framework. Yezzi 2] proposed an active contour approach, but it is only suited to single object well-defined images with relatively large structures. Wyatt 4] used a method that applies Markov random fields in the solution of a MAP model of segmentation and registration. However, it is restricted to rigid registration, and its hard-assignment segmentation makes it vulnerable to noise.

In this paper, we present a novel MAP model for simultaneous segmentation and registration (SSR). We include a hidden Markov random vector field into the model to improve the performance of the segmentation method. We also incorporate B-spline based free-form deformation (FFD) to cope with the nonrigid registration. 


\section{Method}

We have two images $I$ and $J$, and we assume $J$ corresponds to some unknown geometric transformation of reference image $I$. "Segmentation", or the labelling of each pixel to one tissue type can be regarded as a model of the underlying anatomy. $I$ and $J$ can be interpreted as a realization of a random process that corrupts the "Segmentation", e.g. by Gaussian noise. The problem can be formulated as follows: given image $I$ and $J$, we wish to simultaneously estimate the label fields $f$ of the images and recover the geometric transformation $T$ that registers the two images. The MAP estimation is to find $f$ and $T$ to maximize $P(f, T \mid I, J)$.

\subsection{Hidden Vector Field}

We assume that there are $M$ regions; discrete label $f(\boldsymbol{r})$ indicates to which region pixel $\boldsymbol{r}=\left(x_{r}, y_{r}\right)$ belongs. As noted above, the relationship between an image and its segmentation can be defined in terms of a Gaussian noise distribution:

$$
P(I \mid f)=\prod_{\boldsymbol{r} \in \Omega_{I}} v_{f(\boldsymbol{r})}(\boldsymbol{r}) .
$$

where $\Omega_{I}$ is the lattice of sites of $I$ and each $M$-vector $\boldsymbol{v}(\boldsymbol{r})$ is defined by:

$$
v_{k}(\boldsymbol{r})=\sqrt{\frac{\gamma}{\pi}} \exp \left[-\gamma\left|I(\boldsymbol{r})-\theta_{k}\right|^{2}\right] .
$$

Here, $\gamma$ and $\boldsymbol{\theta}$ are image parameters that depend on the noise variance and mean intensity value of each class. One can find the optional estimate for $f$ by applying a classical MRF model and Bayesian MAP estimation [5].

Marroquin [3] proposed a different probabilistic model for the generation of label field to overcome the difficulties with classical MRF models like sensitive to noise and initialization. Instead of the conventional 1-step procedure, he proposed a 2-step probabilistic model, with an additional hidden Markov random vector field $p$ : each vector $\boldsymbol{p}(\boldsymbol{r})$ indicates the probability the pixel $\boldsymbol{r}$ belongs to one of the regions given the intensity of that pixel, and it takes values on the $M$-vertex simplex $S_{M}$ :

$$
S_{M}=\left\{\boldsymbol{u} \in \mathcal{R}^{M}: \sum_{k=1}^{M} u_{k}=1, u_{k} \geq 0, k=1, \ldots, M\right\} .
$$

Then, the optimal estimate for $f$ can be calculated from $p$.

\subsection{General Framework}

We incorporate the Markov random vector field $p$ into our framework. To obtain the optimal estimator $f^{*}$ for the label field and $T^{*}$ for transformation, we follow the steps: 
1. Find the MAP estimators $p^{*}, T^{*}$ for $p, T: p^{*}, T^{*}=\arg \max _{p \in S_{M}^{N}, T}$ $P(p, T \mid I, J)$

2. Determine $f^{*}(\boldsymbol{r})=\arg \max _{f(\boldsymbol{r})} P\left(f \mid p=p^{*}, I\right)=\arg \max _{k} p_{k}^{*}(r)$

The first step itself is a 2-step procedure, in which the best $T$ is found given the current estimate for $p$, then the best estimate for $p$ is found, given the current estimate for transformation $T$ :

1. Find an initial estimate $\bar{p}$ for $p$ by individual segmentation;

2. Repeat until convergence or often enough:

(a): Set $\bar{T}=\arg \max _{T} P(T \mid \bar{p}, I, J)$

(b): Set $\bar{p}=\arg \max _{p} P(p \mid \bar{T}, I, J)$

We now analyze step (a). We consider image $I$ to be the reference image, transformation $T$ to be a spatial mapping from $I$ to $J$. Using Bayes' rule, we have:

$$
P(T \mid \bar{p}, I, J) \propto P(I \mid \bar{p}) P(J \mid \bar{p}, T) P(T) .
$$

In order to maintain spatial coherence and smoothness, the transformation $T(\boldsymbol{r})$ may be required to be similar to its value at the spatial neighbors. We assume a Gibbs distribution on the expected deformations: $P(T)=\exp (-E(T))$, where $E(T)$ is $T$ in the form of an energy. The likelihood of the observations can be rewritten:

$$
P(J \mid \bar{p}, T)=\prod_{\boldsymbol{r} \in \Omega_{I}} P(J(T(\boldsymbol{r})) \mid \bar{p}) .
$$

For a Gaussian noise distribution, we have:

$$
p(J(T(\boldsymbol{r})) \mid \bar{p})=\sum_{k=1}^{M} w_{k}(T(\boldsymbol{r})) \bar{p}_{k}(\boldsymbol{r})=\boldsymbol{w}(T(\boldsymbol{r})) \cdot \overline{\boldsymbol{p}}(\boldsymbol{r}) .
$$

where

$$
\left.w_{k}(T(\boldsymbol{r}))=\left.\sqrt{\frac{\gamma}{\pi}} \exp \left[-\gamma \mid J(T(\boldsymbol{r}))-\theta_{k}\right)\right|^{2}\right] .
$$

Finally we get:

$$
P(T \mid \bar{p}, I, J) \propto \exp [-U(T)] .
$$

where

$$
U(T)=-\sum_{\boldsymbol{r} \in \Omega_{I}} \log (\boldsymbol{w}(T(\boldsymbol{r})) \cdot \overline{\boldsymbol{p}}(\boldsymbol{r}))+E(T) .
$$

Step (a) is equivalent to minimizing of $U(T)$.

For step (b):

$$
P(p \mid \bar{T}, I, J) \propto P(I \mid p) P(J \mid p, \bar{T}) P(p) .
$$

Since $p$ is Markovian, $P(p)$ can be expressed as $P(p)=\exp (-V(p))$, where, for example,

$$
V_{\boldsymbol{r} \boldsymbol{s}}(\boldsymbol{p}(\boldsymbol{r}), \boldsymbol{p}(\boldsymbol{s}))=\lambda|\boldsymbol{p}(\boldsymbol{r})-\boldsymbol{p}(\boldsymbol{s})|^{2}=\lambda \sum_{k=1}^{M}\left(p_{k}(\boldsymbol{r})-p_{k}(\boldsymbol{s})\right)^{2} .
$$

where $\lambda$ is a positive parameter, and $\langle\boldsymbol{r}, \boldsymbol{s}\rangle$ are neighboring sites in $\Omega_{I}$. 
So, we get:

$$
P(p \mid \bar{T}, I, J) \propto \exp [-U(p)]
$$

with

$$
U(p)=-\sum_{\boldsymbol{r} \in \Omega_{I}} \log (\boldsymbol{v}(\boldsymbol{r}) \cdot \boldsymbol{p}(\boldsymbol{r}))-\sum_{\boldsymbol{r} \in \Omega_{I}} \log (\boldsymbol{w}(\bar{T}(\boldsymbol{r})) \cdot \boldsymbol{p}(\boldsymbol{r}))+\sum_{C} V_{C}(p) .
$$

Step (b) is then equivalent to minimizing of energy $U(p)$. we use iterative gradient descent method for optimization of both $T$ and $p$. Here, $\boldsymbol{p}(\boldsymbol{r})$ must be projected back into $S_{M}$.

\subsection{The Representation of Transformation}

For the case where $T$ is a rigid transformation, we represent it by a rotation matrix $A$ and a translation vector $\boldsymbol{c}: T(\boldsymbol{r})=A \boldsymbol{r}+\boldsymbol{c}$. In two dimensions, the rotation matrix $A$ depends upon a single angle $\alpha$. Since for a rigid registration, all the pixels undergo the same transformation, it is sufficiently smooth for $E(T)$ to be dropped, so we can set it to zero. By minimizing $U(T)$, we get parameters $\alpha, \boldsymbol{c}$ to represent $\bar{T}$.

For non-rigid transformation, we represent $T$ using a combination of a global transformation and a local transformation:

$$
T=T_{\text {global }}+T_{\text {local }}
$$

The global transformation is represented by a rigid transformation, while for and the local transformation, we use a B-spline based FFD model [1]:

$$
T_{\text {local }}(\boldsymbol{r})=\sum_{m=0}^{3} \sum_{n=0}^{3} B_{m}(u) B_{n}(v) \Phi_{i+m, j+n} .
$$

where $\Phi$ denotes lattice of control points, $i, j$ denote the indices of the control points and $u, v$ correspond to the relative positions of $\boldsymbol{r}$ in lattice coordinates. The lattice of control points is defined as a grid with uniform spacing which is placed on the underlying reference image.

For $T_{\text {global }}$, the $E(T)$ can be dropped, and for $T_{\text {local }}$, we may use a 5 pixels neighborhood clique $C: E(T)=\sum_{C} V_{C}(T)$ where

$$
\begin{aligned}
\sum_{C} V_{C}(T(\boldsymbol{r})) & =2\left(T\left(x_{r}, y_{r}+1\right)+T\left(x_{r}-1, y_{r}\right)-T\left(x_{r}, y_{r}\right)-T\left(x_{r}-1, y_{r}+1\right)\right)^{2} \\
& +\left(T\left(x_{r}-1, y_{r}\right)+T\left(x_{r}+1, y_{r}\right)-2 T\left(x_{r}, y_{r}\right)\right)^{2} \\
& +\left(T\left(x_{r}, y_{r}-1\right)+T\left(x_{r}, y_{r}+1\right)-2 T\left(x_{r}, y_{r}\right)\right)^{2}
\end{aligned}
$$




\section{$3 \quad$ Experiments}

In this section, we use brain MR images to illustrate the performance of our approach of simultaneous segmentation and registration presented above.

In order to compare the algorithms, we need to establish a performance index, which should be objective and quantitative. We propose the following performance index $\xi$ :

$$
\xi_{k}=\frac{2 V_{G P k}}{V_{P k}+V_{G k}}
$$

where $V_{G P k}$ denotes the total number of pixels that were correctly assigned to class $k$ by a given procedure; $V_{P k}$ is the total (correct + incorrect) number of pixels belonging to class $k$ by this procedure and $V_{G k}$ denotes the total number of pixels belonging to class $k$ in the ground truth. Higher performance index indicates better segmentation result here.

Our initial experiments are on brain MR images provided by Brainweb [6]. We segment the images into 3 tissue classes in the brain: cerebrospinal fluid (CSF), gray matter (GM), and white matter (WM). Our first experiment is on images with known rigid transformation between them. We make a transformation on zero noise brain image with 5.00 degree rotation and 11.0 pixels translations in both directions. Various amount of zero-mean Gaussian white noise are independently added to the original and transformed images to produce the observed images $I$ and $J$. The test images, together with their single and SSR results are shown in Fig. 1] We compare these two results with our performance index: as can be seen from Table 1, in each tissue class, the performance index of SSR is always higher than that of the single segmentation. The recovered transformation is 5.03 degree and the translations in two direction are 10.7 and 10.4 pixels.

Our second experiment is on images with an unknown non-rigid transformation: we take two different slices of brain MRI as $I$ and $J$; the image experimental results are shown in Fig. 2. We can see from the performance index in Table 2 that the SSR gives more correct classification for pixels in each class than the single segmentation. The recovered transformation is represented by transforming the floating image into the reference image domain, since no real transformation ground truth can be provided here, we can only get a visually qualitative impression of our registration result. We aim to study how to evaluate non-rigid registration methods later.

Table 1. Performance index comparison between single and SSR segmentation for experiment1

\begin{tabular}{llll}
\hline tissue class & CSF & GM & WM \\
\hline single & 0.893 & 0.869 & 0.930 \\
SSR & 0.894 & 0.874 & 0.937 \\
\hline
\end{tabular}




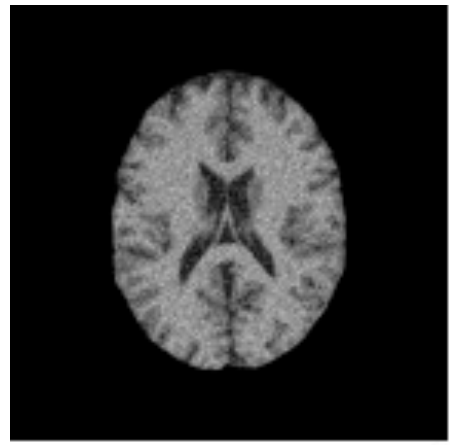

(a)

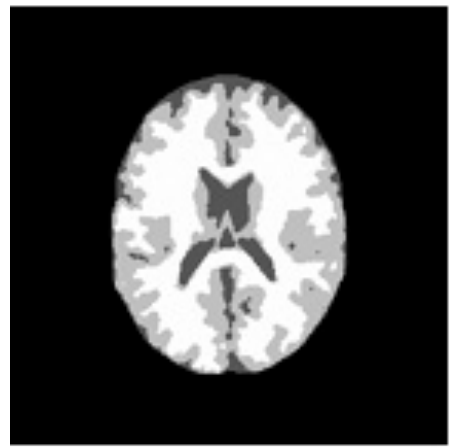

(c)

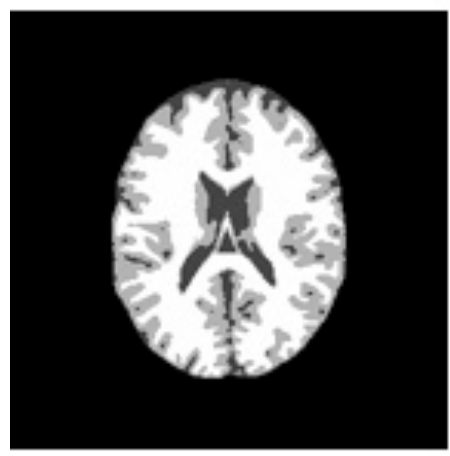

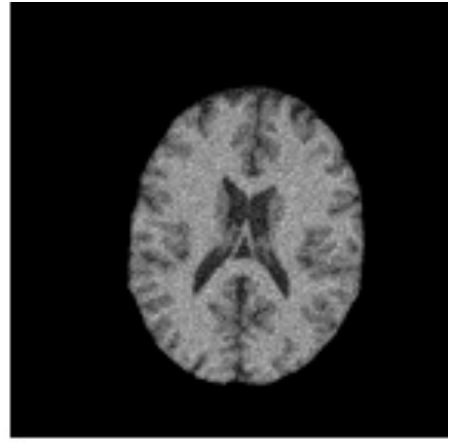

(b)
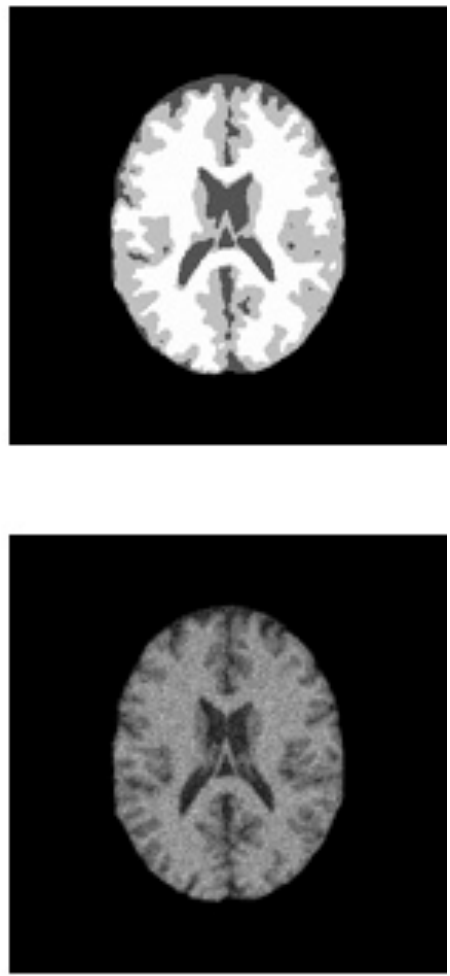

(f)

Fig. 1. Simultaneous segmentation and registration (SSR) experiment on rigid registration. (a)reference image I. (b)floating image J. (c)segmentation result of image I without SSR. (d)segmentation result with SSR. (e)segmentation ground truth in image I domain. (f)transformation of floating image to reference image using rigid registration. 


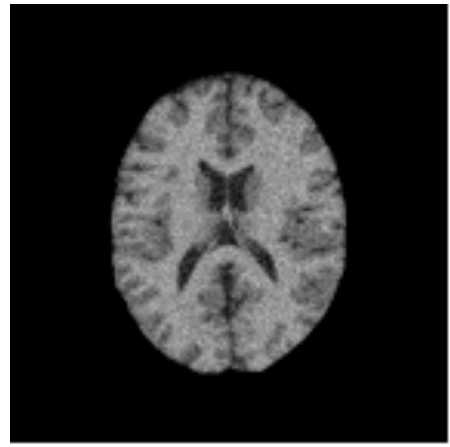

(a)

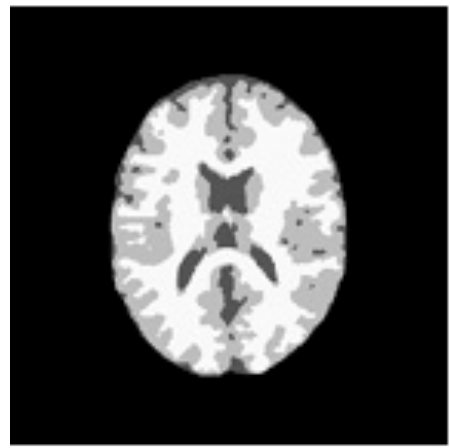

(c)

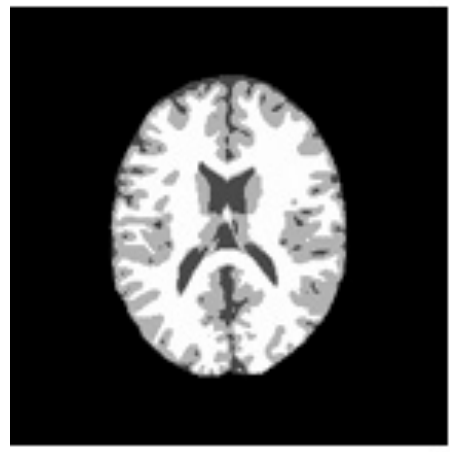

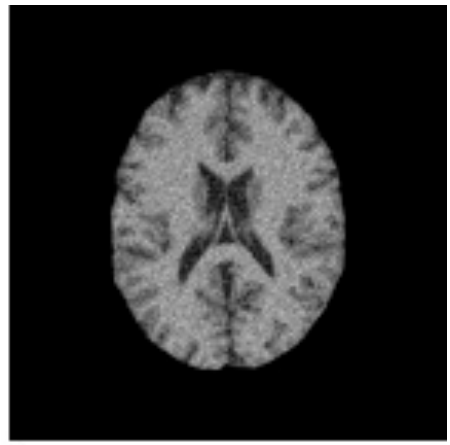

(b)
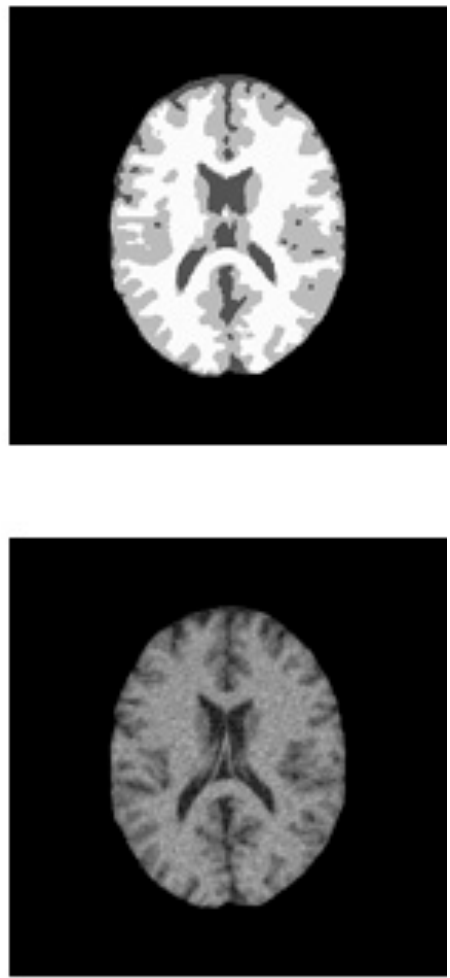

(f)

Fig. 2. Simultaneous segmentation and registration (SSR) on non-rigid registration. (a)reference image I. (b)floating image J. (c)segmentation result of image I without SSR.(d)segmentation result with SSR. (e)segmentation ground truth in image I domain. (f)transformation of floating image to reference image using non-rigid registration. 
Table 2. Performance index comparison between single and SSR segmentation for experiment2

\begin{tabular}{llll}
\hline tissue class & CSF & GM & WM \\
\hline single & 0.882 & 0.888 & 0.939 \\
SSR & 0.890 & 0.892 & 0.942 \\
\hline
\end{tabular}

\section{Summary}

In this paper, we have developed a framework to achieve simultaneously segmentation and registration to make the two problems' solutions facilitate each other. We use a hidden Markov measure vector field to make the interactions between these two problems possible. It is used for segmentation step to label each pixel with highest probability of certain tissue type and for registration step to act as a key element in similarity measure. For reason of space, we have only illustrated this framework to brain MR images for both rigid and non-rigid registration cases, with promising results for both segmentation and registration: the segmentation results achieved by fusion of the images performs better than the segmentation results got from single image, meanwhile, using the segmentation results also produces a good registration.

\section{References}

1. Rueckert, D., Sonoda, L.I., Hayes, C., Hill, D.L.G., Leach, M.O., Hawkes, D.J.: Nonrigid Registration Using Free-Form Deformations: Application to Breast MR Images. IEEE Trans. Medical Imaging, Vol. 18. Num. 8. (1999) 712-721

2. Yezzi, A., Zollei, L.: A Variational Framework for Integrating Segmentation and Registration Through Active Contours. Medical Image Analysis, Vol. 7. (2003) 171185

3. Marroquin, J.L., Santana, E.A., Botello, S.: Hidden Markov Measure Field Models for Image Segmentation. IEEE Trans. Pattern Analysis and Machine Intelligence, Vol. 25. Num. 11. (2003) 1380-1387

4. Wyatt, P.P., Noble, J.A.: MAP MRF Joint Segmentation and Registration of Medical Images. Medical Image Analysis, Vol. 7. Num. 4. (2003) 539-552

5. Zhang, Y., Brady, M., Smith, S.: Segmentation of Brain MR Images Through a Hidden Markov Random Field Model and the Expectation Maximization Algorithm. IEEE Trans. Medical Imaging, Vol. 20. Num. 1. (2001) 45-57

6. Cocosco, C.A., Kollokian, V., Kwan, R., Evans, A.C.: BrainWeb: Onlie Interface to a 3D MRI Simulated Brain Database. Neuroimage, Vol. 5. Num. 4. (1997) 425 